\title{
Dietary niche breadth influences the effects of urbanization on the gut microbiota of sympatric rodents
}

\author{
Jason Anders ${ }^{1}$, Alexis Mychajliw ${ }^{2}$, Mohamed Moustafa ${ }^{1}$, Wessam Mohamed ${ }^{1}$, Takashi \\ Hayakawa $^{1}$, Ryo Nakao ${ }^{1}$, and Itsuro Koizumi ${ }^{1}$ \\ ${ }^{1}$ Hokkaido University \\ ${ }^{2}$ Middlebury College
}

July 28, 2021

\begin{abstract}
Cities are among the most extreme forms of anthropogenic ecosystem modification and urbanization processes exert profound effects on animal populations through multiple ecological pathways. Increased access to human associated food items may alter species' foraging behavior and diet, in turn modifying the normal microbial community of the gastrointestinal tract, ultimately impacting their health. It is crucial we understand the role of dietary niche breadth and the resulting shift in the gut microbiota as urban animals navigate novel dietary resources. We combined stable isotope analysis of hair and microbiome analysis of four gut regions across the gastrointestinal tract to investigate the effects of urbanization on the diet and gut microbiota of two sympatric species of rodent with different dietary niches; the omnivorous large Japanese field mouse (Apodemus speciosus) and the relatively more herbivorous grey red-backed vole (Myodes rufocanus). Both species exhibited an expanded dietary niche width within the urban areas potentially attributable to novel anthropogenic foods and altered resource availability. We detected a dietary shift in which urban A. speciosus consumed more terrestrial animal protein and M. rufocanus more plant leaves and stems. Such changes in resource use may be associated with an altered gut microbial community structure. There was an increased abundance of the presumably probiotic Lactobacillus in the small intestine of urban A. speciosus and potentially pathogenic Helicobacter in the colon of M. rufocanus. Together, these results suggest that even taxonomically similar species may exhibit divergent responses to urbanization with consequences for the gut microbiota and broader ecological interactions.
\end{abstract}

\section{Introduction}

We live in an increasingly urbanized world and the formation of cities is accompanied by ongoing shifts in the ecology and life history of wildlife that inhabit these areas, which now span some of the most biodiverse regions on the planet. (Güneralp et al., 2020; Seto et al., 2012; Shochat et al., 2006). Urbanization can negatively impact the health and survival of animals (Murray et al., 2019) through habitat fragmentation (Beninde et al., 2015; Faeth et al., 2011), artificial feeding (Murray et al., 2015), and pollution (Isaksson, 2015). Importantly, the high degree of human-wildlife interactions within urban areas not only alters animal behavior (Bateman \& Fleming, 2014; Ditchkoff et al., 2006) but can also increases the transmission risk of zoonotic parasites and diseases such as Echinococcus , hantavirus, and Lyme disease (Bradley \& Altizer, 2006; Mackenstedt et al., 2014). Therefore, fostering conditions that support biodiverse ecosystems is important not only from a conservation point of view for individual wildlife species, but also extends into public health discourse (i.e. One Health; Destoumieux-Garzón et al., 2018).

One area of recent but rapidly growing interest is how urbanization affects the gut microbiota of wildlife (Fuirst et al., 2018; Littleford-Colquhoun et al., 2019; Murray et al., 2020; Phillips et al., 2018; Sugden et al., 2020; Teyssier et al., 2018, 2020). The gut microbial communities of animals play a pivotal role in development (Fraune \& Bosch, 2010), nutritional uptake (Hooper et al., 2002), and general immune system function 
(Schluter et al., 2020). Therefore, any disruption to the gut microbial community (a condition known as dysbiosis) can adversely impact numerous aspects of the host's physiology and life history, ultimately affecting their health and survival (Logan et al., 2016). For example, diet simplification due to forest fragmentation can decrease gut microbial diversity (Amato et al., 2013) and negatively affect immune system function (de Paiva et al., 2016). Dietary shifts in urban animals resulting from artificial feeding especially of low quality foods (Murray et al., 2015), may cause a detrimental shift in the microbial community structure within the gut that can induce numerous health issues such as obesity or inflammatory bowel disease (Chin et al., 2000; Singh et al., 2017). Furthermore, urbanization can lead to higher levels of both acute and chronic stress due to chemical, noise, and light pollution that can alter the gut microbiota and negatively impact host health (Gao et al., 2018; Isaksson, 2015).

A handful of recent studies have shown changes in the gut microbial communities of urban animals as compared to conspecifics in less urbanized environments, but the degree and directions were equivocal among host taxonomic groups and study area (Fuirst et al., 2018; Littleford-Colquhoun et al., 2019; Murray et al., 2020; Phillips et al., 2018; Sugden et al., 2020; Teyssier et al., 2018, 2020). Importantly, no study has compared the gut microbial communities between sympatric species with different dietary niches within the same urban environments. Because dietary habits determine the likelihood of utilizing novel urban resources (Pagani-Núñez et al., 2019) in turn affecting the gut microbiota and impacting host adaptability to highly modified environments, understanding the role of dietary niche breadth is crucial for predicting the effects of urbanization on the gut microbiota. We expected that dietary generalists are more likely to flexibly shift their diet in urban environments, thereby strongly altering their gut microbiota more so than species with a more restrictive diet.

We investigated the gut microbial community in four regions (small intestine, cecum, colon, and rectum) of the gastrointestinal tract (GIT) of two sympatric species of rodent in urban areas as compared to conspecifics in more natural environments (minimally modified / developed). Specifically, we were interested if a difference in dietary niche would cause a differential response to novel anthropogenic food resources that could be linked to changes in the gut microbiota. The large Japanese field mouse (Apodemus speciosus) and the grey redbacked vole (Myodes rufocanus ) belong to the same taxonomic family Muridae and occupy the same habitat patches throughout the island of Hokkaido, Japan (Kaneko et al., 1998; Saitoh et al., 2007). While both species are omnivorous, A. speciosus preferentially consumes seeds, nuts, and insects, while M. rufocanus is more restricted with a diet predominantly composed of herbaceous plants (Kaneko et al., 1998; Tatsukawa \& Murakami, 1976). We predicted that urban A. speciosus populations would have a more expanded dietary niche width than $M$. rufocanus as compared to conspecifics in more natural habitats. This expansion should have a positive effect on gut microbiome alpha diversity in all four regions of the GIT as compared to natural conspecifics. We expected the largest impact in the lower GIT (i.e. cecum, colon, and rectum) where fermentation of plant polysaccharides occurs and the host immune system has a diminished role in shaping the microbial community (Donaldson et al., 2016). Furthermore, because novel food items are likely to require a shift in the digestive capabilities of the microbial flora, we expected urban A. speciosus to exhibit a larger change in the microbiota (Carmody et al., 2015; David et al., 2014). Even if the general category of dietary source (i.e. insects or plants) does not change in the urban environment, the specific plant and insect species consumed may (Beninde et al., 2015; Faeth et al., 2011). This study aimed to generate generalized insights into the conditions that lead to dietary changes within the urban environment and the subsequent response of the gut microbiota, ultimately impacting the adaptability of animals to highly modified environments.

\section{Methods}

\section{Fieldwork and gut content sampling}

In October 2019, two sympatric species of rodent, the large Japanese field mouse (A. speciosus ), and the grey red-backed vole ( $M$. rufocanus ) were captured in forest fragments within two urban parks (i.e. Kaguraoka koen and Shunkodai koen) in the city of Asahikawa and one park (i.e. Maruyama koen) in the city of Biei, as well as four natural sites (i.e. Shirakkeyama, Chitoseyama, Harushinai, and Mukoyama) within the surrounding Kamikawa Chubu National forest in central Hokkaido, Japan (Fig. S1, Table S1). The urban 
parks are surrounded by residential areas with Kaguraoka koen notably situated next to the central built-up area of Asahikawa city. All parks are actively managed and heavily used by the public. Our natural sites were located in the middle of the forest at least $1 \mathrm{~km}$ from any agricultural or built-up areas preventing exposure to human impacts as the species home ranges are much smaller (Ims, 1987; Oka, 1992). All natural sites were higher in elevation than any potential pollution runoff but below $500 \mathrm{~m}$ in order to avoid altitudinal variation in the gut microbiome (Suzuki et al., 2019). Both the national forest and managed forest fragments within the urban parks were primarily composed of deciduous trees such as birch, oak, and walnut while the underbrush was mostly dwarf bamboo (Sasa kurilensis ) with some various small leafy plants. At each site, we deployed two or three trap grids of Sherman traps (H.B. Sherman Traps, Inc.) baited with oatmeal and placed in a 4x10 grid pattern with each trap 10m apart. Traps were checked within one hour after sunrise for two to three consecutive days, and any trap containing an animal was replaced with a fresh one.

Animals were transported to the Department of Parasitology at Asahikawa Medical University in Asahikawa where they were euthanized and identified. Body condition was determined by dividing the log of body weight by the log of body length (Labocha et al., 2014). The gastrointestinal tract was removed, and gut content was collected from the ileum in the small intestine, the cecum, and the descending colon, as well as fecal matter from the rectum using a small steel spatula and utilizing sterilization-based laboratory techniques. Samples were placed in a $-80^{\circ} \mathrm{C}$ freezer within 1 hour of collection where they were stored until transfer to the Laboratory of Parasitology in the Faculty of Veterinary Medicine at Hokkaido University, Sapporo, Japan for DNA processing. Finally, hair was collected from the outer hind legs and dried under a fume hood for 48 hours for use in stable isotope analysis. Experimental design and handling of animals was approved by the Institutional Animal Care and Use Committee of the national University Corporation Hokkaido University (reference number 15-0121) and carried out in accordance with their guidelines. This study was conducted in compliance with the ARRIVE guidelines 2.0.

\section{Stable isotope analysis}

We utilized stable isotopes of carbon $\left(\delta^{13} \mathrm{C}\right)$ and nitrogen $\left(\delta^{15} \mathrm{~N}\right)$ to reconstruct the diet of our target host species, a widely applied technique in ecological studies (Baltensperger et al., 2015; Ben-David \& Flaherty, 2012). Here, we analyzed stable isotope values of hair as a proxy for long term resource use to determine the effect of dietary habits on the gut microbial community. Before analysis, hair was washed using a chloroform:methanol solution $(2: 1 \mathrm{v} / \mathrm{v})$ for removal of surface oils, then rinsed in distilled water and dried in an oven at $60^{\circ} \mathrm{C}$ for 48 hours. We wrapped $0.5 \mathrm{mg}$ of hair from each individual in tin capsules and analyzed stable isotope ratios using an elemental analyzer (Flash EA 1112, Thermofisher) coupled to an isotopic ratio mass spectrometer (IRMS, Delta V Plus, Thermofisher). Standardization of isotopic ratios are based on Vienna Pee Dee Belemnite (VPDB) for $\delta^{13} \mathrm{C}$ and atmospheric nitrogen (AIR) for $\delta^{15} \mathrm{~N}$ and presented as parts per mil (

\section{Extraction, PCR, and high-throughput sequencing}

Following Hayakawa, et al. (2018), DNA was extracted from gut content and fecal matter using the QIAamp fast DNA stool mini kit (Qiagen) after bead beading for three minutes with $1 \mathrm{mg}$ of $0.1 \mathrm{~mm}$ and four $3 \mathrm{~mm}$ silica/zirconia beads. PCR amplification of the V3-V4 region of the 16S rRNA gene was performed using the $314 \mathrm{~F} / 805 \mathrm{R}$ universal primers (Klindworth et al., 2013). DNA extraction and PCR amplification were done under sterile conditions and a negative control was included in each batch of samples. Finally, highthroughput sequencing was done on a MiSeq (Illumina, San Diego, USA) 301bp paired-end platform using a Reagent kit v3 after library preparation using Nextera XT Index Kit v2 set A, B, C, or D following the manufacturer's instructions. A more detailed description of the DNA processing methods can be found in the supplementary materials and raw sequences have been submitted to the DNA database of Japan (DDBJ) with the accession numbers DRA011343 and DRA011772.

\section{Data analysis}

We used a pairwise Wilcoxon rank-sum test in the statistical program $\mathrm{R}$ (version 4.0.2; R Core Team, 2020) to compare $\delta^{13} \mathrm{C}$ and $\delta^{15} \mathrm{~N}$ values between species and habitat conditions as the data deviated from normality. 
We then investigated the $\delta^{13} \mathrm{C}$ and $\delta^{15} \mathrm{~N}$ isotopic niche space occupied by urban and natural populations by calculating standard ellipse area (small sample size corrected; $\mathrm{SEA}_{\mathrm{c}}$ ) for each host species in the $\mathrm{R}$ package SIBER (Jackson et al., 2011). A Bayesian multivariate distribution was fit to each host species in urban and natural habitats using Gibbs sampling technique over 20,000 iterations with a burn in of 1000 implemented in the R package rjags (Plummer, 2019). We compared the niche size of urban and natural populations using maximum likelihood estimates of $\mathrm{SEA}_{\mathrm{c}}$ and by calculating the posterior distribution of the covariance matrix generating Bayesian SEA $\left(\mathrm{SEA}_{\mathrm{b}}\right)$. For pairwise comparisons between the ellipse sizes of the different species and ecosystems, the probability that a given ellipse had a larger posterior distribution was calculated using paired posterior draws where the proportion of draws that were larger serves as a proxy for probability. We also calculated the proportional overlap of maximum likelihood fitted standard ellipses to quantify the degree of niche space overlap between the two species or habitat type, where 0 indicates no overlap and 1 complete overlap.

We quantified the differential contributions of food resource categories to both species in each habitat type using a stable isotope mixing model in the R package SIMMR (Parnell, 2020). Because no specific trophic enrichment factors are available for these rodent species, we used an average value of rodent hair isotopic offsets $\left(\delta^{15} \mathrm{~N} 2.7 \pm 1.67\right.$ study of similar focal taxa (i.e. rodents; Baltensperger et al., 2015). Isotopic values of potential food items were taken from previously published studies in Hokkaido including various C3 plant leaves and stems $\left(\delta^{13} \mathrm{C}-31.65 \pm 0.62-1.98 \pm 1.32\left(\delta^{13} \mathrm{C}-28.15 \pm 1.12 \pm 0.330 .042014\right)\right.$, and terrestrial animals including herbivorous mammals and insects $\left(\delta^{13} \mathrm{C}-26.3 \pm 0.51 .5 \delta^{13} \mathrm{C}\right.$ values were corrected for the Suess effect of atmospheric carbon depletion using a year-specific correction value to 2019 values (Long et al., 2005). Model fit was evaluated through assessment of Gelman diagnostics of MCMC convergence and a posterior predictive check.

Paired-end sequence reads of the microbiome from gut content and fecal matter were demultiplexed, merged, denoised, and quality filtered using the default DADA2 pipeline in QIIME2 version 2020.2 (Bolyen et al., 2019; Callahan et al., 2016), producing a table of amplicon sequence variants (ASVs). Few sequence reads were found within our negative controls (Average $=130 \pm 168.98 \mathrm{SD}$ ), thereby limiting their use for decontaminating our samples. Therefore, potential contaminant sequence reads were identified using the frequency method with a threshold of 0.1 in the decontam package in R (Davis et al., 2018). A total of 195 potential contaminant sequence reads were identified and the frequency plots of each were checked for confirmation. We then manually confirmed the presence of each potential contaminant within our negative controls. Four sequence reads were found to be highly prevalent and highly abundant within our samples yet rare or non-existent within our negative controls. Therefore, a total of 191 potential contaminant sequences were removed using sequence identifiers in QIIME2. The SILVA classifier (release 138) was used for taxonomic classification of each ASV (Bokulich et al., 2018). Only bacterial ASVs identified at phylum level or below were kept for further analysis; all others were removed. A rooted phylogenetic tree was generated using the FastTree method with the MAFFT plugin in QIIME2 (Price et al., 2010).

Based on alpha rarefaction analysis, samples were rarified to a sampling depth of 10,000 reads to calculate diversity metrics. A total of six samples (three small intestine and one feces from M. rufocanusand two small intestine from A. speciosus ) with low sequence counts were excluded from diversity analysis. Alpha diversity was quantified using Shannon's Diversity, Faith's phylogenetic diversity (PD), Pielou's evenness, and the number of ASVs. To analyze beta-diversity comparing habitat type, unweighted and weighted UniFrac dissimilarity matrices were generated in Qiime2.

We investigated whether alpha diversity of the gut microbiome of rodents is altered within the urban environment by developing a linear mixed effects model (LME) in which the response variable was log transformed alpha diversity, the random effect was site, and the explanatory variables were habitat type (i.e. urban or natural), sex, age (adult or sub-adult determined by weight), $\delta^{13} \mathrm{C}$, and $\delta^{15} \mathrm{~N}$ using the nlme package in $\mathrm{R}$ (Pinheiro et al., 2020). This was repeated for each gut region in each host species. Body condition was not included in the models because we found no difference between urban and natural populations in neither $A$. speciosus(ANOVA, $\mathrm{F}=0.186, p=0.667$ ) nor $M$. rufocanus (ANOVA, $\mathrm{F}=0.333, p=0.565$ ). Beta-diversity 
was first visualized using principle coordinate analysis ( $\mathrm{PCoA}$ ) plots for each gut region in each species performed in the R package phyloseq (McMurdie \& Holmes, 2013). PERMANOVA analysis was then conducted to determine the impact of habitat type, sex, age, $\delta^{13} \mathrm{C}$, and $\delta^{15} \mathrm{~N}$ on beta-diversity within each gut region of both rodent species using the adonis2 function with the margin=" by" option to determine the marginal effect of each in the vegan package in R (Oksanen et al., 2020). Within habitat type variation of the gut microbial community within each gut region was analyzed using PERMDISP with 999 permutations in the vegan package in $\mathrm{R}$ for both unweighted and weighted UniFrac dissimilarity metrics (Oksanen et al., 2020).

Linear discriminant analysis effect size (LEfSe) was used to compare relative abundances of the different microbial genera between urban and natural populations of both A. speciosus and M. rufocanus. This was done for each gut region separately within each species using the Huttenhower lab Galaxy pipeline where class was habitat type (Segata et al., 2011). We selected those genera identified as being significantly more abundant in either the urban or natural habitats with either known probiotic or pathogenic species, or to have an association with a high protein diet to further explore what variables may be influencing their abundances. We used a generalized linear mixed effects model (GLMM) with negative binomial distribution to investigate if age, sex, $\delta^{13} \mathrm{C}$, and $\delta^{15} \mathrm{~N}$ (i.e. fixed effect variables) had any effect on their abundances (i.e. response variable) with site as a random effect and habitat type added as an additional fixed effect to confirm the results of LEfSe analysis. The models were run using the NBZIMM package in $\mathrm{R}$ (Zhang et al., 2018). The association between our explanatory variables and relative abundance was then analyzed in natural and urban populations separately to determine potential within ecosystem specific interactions.

\section{Results}

\section{Host capture and gut content}

We captured 42 and 41 A. speciosus, and 43 and $50 \mathrm{M}$. rufocanus from natural and urban habitats respectively (Table S1). A total of 245 gut content and fecal matter samples were collected from A. speciosus consisting of 81 from the small intestine (42 natural, 39 urban), 43 from the cecum (27 natural, 16 urban), 80 from the colon (41 natural, 39 urban), and 41 from the rectum (25 natural, 16 urban). From M. rufocanus , 92 gut content samples were collected from the small intestine (43 natural, 49 urban), 31 from the cecum (14 natural, 17 urban), 83 from the colon (38 natural, 45 urban), as well as 32 rectum samples (16 natural, 16 urban) comprising a total of 238 . After quality filtering, $21,820,759$ high quality reads were obtained with $11,264,730$ (average of $45978 \pm 12453$ SDper sample) from A. speciosus and 10,556,029 (average of $44167 \pm$ 16512 SD per sample) from $M$. rufocanus .

\section{Expanded dietary niche width and dietary shift}

We found significant differences in $\delta^{15} \mathrm{~N}(p<0.001)$ between natural and urban populations of both species, but not $\delta^{13} \mathrm{C}\left(p>0.05\right.$; Fig. 1). There was a significant difference in both $\delta^{13} \mathrm{C}$ and $\delta^{15} \mathrm{~N}$ values between species in the urban areas $(p<0.001)$, but only $\delta^{13} \mathrm{C}$ was significantly different within the natural sites (Fig. 1, Table S2).

Isotopic niche width (standard ellipse area, sample size corrected; $\mathrm{SEA}_{\mathrm{c}}$ ) found that urban A. speciosus $\left(\mathrm{SEA}_{\mathrm{c}}\right.$ $=5.78)$ had an isotopic niche almost twice as large as those in the natural habitat $\left(\mathrm{SEA}_{\mathrm{c}}=2.9\right)$ with a 0.9988 probability of the urban ellipse being larger than the natural based on paired posterior draws of the Bayesian distribution (Figs. 2, S2, Table S3). M. rufocanus also exhibited a larger niche width in the urban parks although to a lesser degree than A. speciosusas expected, with an $\mathrm{SEA}_{\mathrm{c}}$ of 2.68 and 3.01 in the natural and urban areas respectively, and a probability of 0.7138 that the urban ellipse was larger (Figs. 2, S2). Furthermore, the degree of pairwise overlap between natural and urban populations were larger for $M$. rufocanus (0.59) than A. speciosus (0.39). A. speciosus also had larger $\mathrm{SEA}_{\mathrm{b}}$ values than $M$. rufocanus in both the urban (0.9993 probability) and natural (0.6645 probability) habitats.

Estimating the proportion of each food item in the diet of both species of rodent found that terrestrial animal protein made up a marginally larger portion of the diet of M. rufocanus $(32.2 \%)$ than A. speciosus $(28.7 \%)$ within the natural habitat, though this slim margin may be due to the choice of input food sources available 
from the literature (Table 1). A. speciosus shifted towards consuming more terrestrial animal protein within the urban parks (43.2\% of their diet) as compared to their natural conspecifics (28.7\%) with a slight shift away from C3 plant leaves and stems (60.4\% to $52.4 \%$ in natural and urban respectively; Table 1). Urban $M$. rufocanus exhibited the opposite trend with shift towards C3 plants (35.9\% to 60.6\% in natural and urban respectively) while consumption of terrestrial animal protein slightly decreased from $32.2 \%$ to $28.26 \%$ (Table 1). Both species were consuming less $\mathrm{C} 3$ fruits and nuts in the urban parks as compared to their natural conspecifics (Table 1).

\section{No change in Alpha diversity in urban populations}

We found that habitat type had no effect on alpha diversity for any diversity index in any gut region for either host species following our prediction for M. rufocanus but not A. speciosus (all $p>0.05$, Figs. 3, $\mathrm{S} 3$, Tables $\mathrm{S} 4$ to $\mathrm{S} 7$ ). Interestingly, neither $\delta^{13} \mathrm{C}$ nor $\delta^{15} \mathrm{~N}$ significantly impacted gut microbiome alpha diversity in any gut region of the omnivorous $A$. speciosus, despite there being significantly higher $\delta^{15} \mathrm{~N}$ values reflected in the hair of urban individuals (Tables S4 to S7). In the more herbivorous M. rufocanus, $\delta^{13} \mathrm{C}$ had a significantly negative relationship with Faith's PD and the number of ASVs in the cecum and rectum (LME: all $p<0.05$; Table S5, S7), with a negative effect in the colon for Faith's PD (LME: $\mathrm{b}=$ $-0.033 \pm 0.015, p=0.035$, Table S5), and in the small intestine for the number of ASVs (LME: $\mathrm{b}=-0.22 \pm$ $0.077, p=0.006$; Table S7). On the other hand, $\delta^{15} \mathrm{~N}$ had a significantly positive effect on Faith's PD and the number of ASVs in both the colon and rectum (all $p<0.05$, Tables S5, S7). Males had significantly higher microbial alpha diversity in the cecum, colon and rectum of $A$. speciosus, but not in the small intestine nor was there an effect of sex in any gut region in M. rufocanus (Tables S4 to S7). Age, on the other hand, significantly impacted alpha diversity in $M$. rufocanus, particularly in the colon and small intestine, but had no effect in A. speciosus (Tables S4 to S7).

\section{Habitat type impacts gut microbiome beta-diversity}

Visualization of the gut microbial community composition using PCoA plots exhibited a high degree of overlap in the clustering of urban and natural populations in all gut regions in both host species, though not entirely (Figs. 4, 5). Interestingly, the cluster area size of urban individuals was the same size or slightly larger than the cluster of individuals from the natural habitats for the cecum, colon, and rectum of both species for both unweighted and weighted UniFrac (Figs. 4, 5). This was also the case in the small intestine of A. speciosus(Fig. 4), but not M. rufocanus where the cluster area of individuals from the urban parks was smaller than those from the national forest (Figs. 5). This trend was largely unconfirmed by PERMDISP as a significant difference in dispersion was only found in the small intestine of $M$. rufocanus for unweighted UniFrac ( $F=4.027, p=0.039$, Table S8). There was also an inverted relationship between PCoA 1 and PCoA 2 for unweighted UniFrac in the cecum and rectum of urban A. speciosus as compared to their natural conspecifics, but only in the rectum according to weighted UniFrac (Fig. 4).

Using PERMANOVA to test for the effect of habitat type on gut microbiota beta-diversity, we found a significant effect of habitat type in all four gut regions of both $A$. speciosus and $M$. rufocanus for unweighted UniFrac (all $p<0.05$, Tables S9, S10). There was also a significant effect in the colon (PERMANOVA: $\left.\mathrm{R}^{2}=0.038, \mathrm{~F}=3.173, p=0.004\right)$ and small intestine (PERMANOVA: $\left.\mathrm{R}^{2}=0.0299, \mathrm{~F}=2.303, p=0.04\right)$ but not the cecum (PERMANOVA: $\left.\mathrm{R}^{2}=0.024, \mathrm{~F}=1.076, p=0,36\right)$ or rectum (PERMANOVA: $\mathrm{R}^{2}=$ $0.042, \mathrm{~F}=1.768, p=0.078$ ) in A. speciosus based on weighted UniFrac (Table S9). In M. rufocanus there was a significant effect of habitat type in the small intestine (PERMANOVA: $\mathrm{R}^{2}=0.051, \mathrm{~F}=4.519, p=$ 0.009), cecum (PERMANOVA: $\mathrm{R}^{2}=0.068, \mathrm{~F}=2.066, p=0.039$ ) and colon (PERMANOVA: $\mathrm{R}^{2}=0.034$, $\mathrm{F}=2.799, p=0.014$ ), but not the rectum (PERMANOVA: $\mathrm{R}^{2}=0.068, \mathrm{~F}=2.054, p=0.064$ ) according to weighted UniFrac (Table S10). Similar to alpha diversity, $\delta^{13} \mathrm{C}$ and $\delta^{15} \mathrm{~N}$ values had a greater effect on gut microbiome beta-diversity in M. rufocanus than A. speciosus. Specifically, $\delta^{13} \mathrm{C}$ significantly impacted beta-diversity in all four gut regions based on unweighted UniFrac (PERMANOVA: all $p<0.05$ ), but only in the small intestine according to weighted UniFrac (PERMANOVA: $\mathrm{R}^{2}=0.033, \mathrm{~F}=2.919, p=0.03$; Table S10). $\delta^{15} \mathrm{~N}$ also significantly impacted beta-diversity in the cecum, colon, and rectum of $M$. rufocanus based on unweighted UniFrac (all $p<0.05$; Table S10), but no effect in any gut region was found for weighted 
UniFrac (all $p>0.05$; Table S10). In A. speciosus, only in the colon was there a significant effect of $\delta^{13} \mathrm{C}$ on gut microbiome beta-diversity according to unweighted UniFrac alone (PERMANVOA: $\mathrm{R}^{2}=0.022, F$ $=1.769, p=0.005$ ) while $\delta^{15} \mathrm{~N}$ had no effect in any gut region (all $p>0.05$, Table S9). Unlike alpha diversity, sex had a minimal impact on beta-diversity in either host species with it only being significant in the colon of $A$. speciosus as well as the rectum and colon of $M$. rufocanus (all $p<0.05$; Tables S9, S10). Age significantly impacted beta-diversity in the colon of $A$. speciosus for both unweighted (PERMANVOA: $\mathrm{R}^{2}=0.024, \mathrm{~F}=1.933, p=0.001$ ) and weighted UniFrac (PERMANVOA: $\mathrm{R}^{2}=0.049, \mathrm{~F}=4.078, p=$ 0.001; Table S9), while it was significant in the cecum, colon, and small intestine of $M$. rufocanus based on unweighted UniFrac (all $p<0.05$; Tables S9, S10).

\section{Differential relative abundance of bacterial genera}

LEfSe analysis found multiple microbial genera in significantly higher abundance in the urban habitat as compared to the national forest or vice versa, with several particularly noteworthy genera (Tables S11 and S12). The probiotic group Lactobacillus (Liu et al., 2010) in the small intestine, Butyricicoccus (Boesmans et al., 2018) in the cecum and colon, and Bifidobacterium (Meddah et al., 2001) in both the small intestine and colon had significantly higher relative abundance in urban A. speciosus as compared to conspecifics in the natural habitats (Fig. 6, Table S11). There was significantly higher abundance of the potentially pathogenic Helicobacter in the colon (Yang et al., 2013) of urban M. rufocanus, while natural populations had higher abundance of Helicobacter the small intestine (Fig. 6, Table S12). Although LEfSe analysis specifically tests for higher abundance of microbes, the opposite can be inferred. For example, the significantly higher relative abundance of Alistipes in both the cecum and colon in the rectum of $M$. rufocanus from the natural ecosystem means there is lower abundance in the urban parks (Fig. 6, Table S12).

In A. speciosus, only the higher relative abundance ofButyricicoccus in the colon of urban individuals was confirmed (GLMM: $b=1.108 \pm 0.355, p=0.026$ ), whileButyricicoccus in the cecum (GLMM: $: b=0.865$ $\pm 0.602, p=0.211$ ), and Lactobacillus in the small intestine (GLMM: $: b=1.088 \pm 0.887, p=0.274$ ) were not (Table S13). Furthermore, none of the model variables exhibited a similar association with relative abundance of any genus within both the natural and urban habitats. Specifically, $\delta^{15} \mathrm{~N}$ had a significantly positive effect on Lactobacillus abundance in the small intestine of natural populations (GLMM: $\mathrm{b}=0.696$ $\pm 0.163, p<0.001$ ) while $\delta^{13} \mathrm{C}$ had a negative effect in the urban animals (GLMM: $\mathrm{b}=-0.526 \pm 0.223, p=$ 0.025; Table S13). There was also a negative effect of $\delta^{13} \mathrm{C}$ values on Butyricicoccus abundance in the colon (GLMM: $\mathrm{b}=-0.892 \pm 0.725, p=0.029$ ) of natural populations of $A$. speciosus(Table S13), but no effect in the urban individuals (GLMM: $\mathrm{b}=0.233 \pm 0.22, p=0.298$, Table S13). Similarly, in M. rufocanusonly the lower abundance of Helicobacter in the small intestine of urban animals (GLMM: $b=-3.2 \pm 1.13, p=$ 0.037) was confirmed by the GLMM, though higher relative abundance in the colon was nearly significant (GLMM: $b=0.793 \pm 0.314, p=0.053$ ) as well as lower abundance of Alistipes in the cecum (GLMM: $\mathrm{b}=$ $-0.528 \pm 0.216, p=0.058$; Table S14). Only Alistipes was significantly affected by $\delta^{15} \mathrm{~N}$ values in the cecum of $M$. rufocanus in the natural habitat (GLMM: $b=0.446 \pm 0.0 .155, p=0.035$; Table S14).

\section{Discussion}

No previous study has contrasted the response of the gut microbiota of multiple animal species within the same urban areas. (Fuirst et al., 2018; Littleford-Colquhoun et al., 2019; Murray et al., 2020; Phillips et al., 2018; Sugden et al., 2020; Teyssier et al., 2018, 2020). Not only does each animal species harbor their own unique gut microbiota (Kohl et al., 2018), interspecific differences in ecological traits effects their interaction with novel anthropogenic environments and their associated stressors, in turn affecting the gut microbiota. Therefore, such changes are likely to be host species-specific. Our design incorporating two sympatric rodent species with differing life histories and ecological traits in the same urban areas permits us to detect differential responses of the gut microbiota when exposed to the same environmental pressures of urbanization. We found that both species exhibited distinct changes in dietary niche, possibly in response to anthropogenic food resources, and may be a key factor in explaining species-specific changes in their gut microbial communities. 


\section{Increased dietary niche width in urban populations}

We assessed species resource use by stable isotopes of carbon $\left(\delta^{13} \mathrm{C}\right)$ and nitrogen $\left(\delta^{15} \mathrm{~N}\right)$, providing a foundation for interpreting how changes in diet may be mediated by local environmental changes and differences in ecological traits resulting in altered microbial communities. Both species of murid rodents appear to occupy an expanded dietary niche in urban parks compared with natural areas, and as expected, this pattern was more pronounced in the omnivorous A. speciosus (Figs. 2, S2). There was less overlap in the isotopic niche space between natural and urban populations of $A$. speciosus suggesting they are more likely to utilize novel food resources and diverge in dietary habits than the more herbivorous $M$. rufocanus . Results similar to what we found in $A$. speciosus have been reported in several omnivorous species occupying urban habitats (Littleford-Colquhoun et al., 2019; Maureen Murray et al., 2015; Pagani-Núñez et al., 2019).

The increased proportion of animal protein consumed by urban A. speciosus as compared to their conspecifics in the national forest possibly comes from the consumption of human provided animal products. This is because urban sites in this study are heavily used parks by local citizens with barbequing and picnics particularly popular activities during late spring to early autumn. Scraps of meat and other food trash were commonly seen throughout the forest fragments during field surveys and may be opportunistically consumed by $A$. speciosus as an easy energy source (Larson et al., 2020; Pagani-Núñez et al., 2019). However, $A$. speciosus could simply be consuming more insects as artificial lighting such as street and park lamps increase insect susceptibility to predation by birds and rodents (Owens et al., 2020; Yoon et al., 2010). The difference in rodent community structure between the two habitat types may also drive the increased consumption of insects. Specifically, within the national forest, the population size of $A$. speciosus and M. rufocanus were inversely related at each site, but in the urban parks they were more equal in number (Table S1) possibly due to forest fragmentation limiting dispersal even when population density is high (Sato et al., 2014). Therefore, increased inter-specific competition for food resources may push each species to preferentially consume food items for which they are more specialized (i.e. insects for A. speciosus and herbaceous plants for M. rufocanus ). This is in contrast to more free roaming animals such as coyotes and birds that have access to a much wider array of micro-habitats within cities and can more readily take advantage of anthropogenic resources thereby avoiding inter-specific competition (Larson et al., 2020; Pagani-Núñez et al., 2019; Phillips et al., 2018).

\section{No change in gut microbial alpha diversity in urban populations}

Altered diets in urban areas can lead to changes in alpha diversity of the gut microbiome potentially decreasing alpha diversity in response to a simplified or low quality diet (Fuirst et al., 2018; Teyssier et al., 2020), or increasing diversity due to access to more diverse or novel resources (Littleford-Colquhoun et al., 2019; Phillips et al., 2018). Lower alpha diversity of the gut microbial community is typically associated with dysbiosis (Logan et al., 2016), highlighting the need to understand how urbanization effects it. We did not detect a change in gut microbial alpha diversity in any gut region within the urban populations of either rodent species as compared to those in the natural habitat despite the wider dietary niche (Figs. 3, S3, Tables S4 to S7). It is possible that other factors not tested for such as pollution or stress have a large enough negative impact on alpha diversity in these animals to mask any positive effect of diet (Gao et al., 2018; Han et al., 2014; Isaksson, 2015). On the other hand, because our stable isotope analysis was at the population level, a more plausible explanation is that intra-individual dietary diversity remains consistent regardless of habitat type, but that inter-individual variation is much greater in the urban parks due to access to novel human associated food items.

\section{Microbial community composition is affected by both diet and habitat type}

Unlike alpha diversity, gut microbial beta-diversity was highly affected by habitat type throughout the GIT of both rodent species (Tables S9, S10). Because PERMDISP did not confirm the larger cluster area of urban populations in the PCoA plots, shifts in the gut microbial community composition are likely at the population level rather than an individual response to the urban environment. Diet is one of the major factors affecting gut microbial community structure (David et al., 2014). The dietary shift of urban populations in 
the present study is a likely factor affecting beta-diversity and overall microbial community structure as $\delta^{13} \mathrm{C}$ and $\delta^{15} \mathrm{~N}$ values had a large impact throughout the GIT of $M$. rufocanus as well as in the colon of A. speciosus (Tables S11, S12). For example,Bifidobacterium was only found in the lower GIT of urban A. speciosus and was non-existent in individuals from the national forest and could be a human associated microbe (Fig. 6, Table S11).Bifidobacterium is typically found in fermented foods such as natto, miso, and yogurt (Fujisawa et al., 2006) that could be consumed by A. speciosus. In M. rufocanus, we found lower abundance of Alistipes in the cecum of urban individuals (Fig. 6, Table S12). This genus is associated with the consumption of animal protein (David et al., 2014), and their lower abundance fits with the shift in diet away from terrestrial animals, although the shift is small and the change in relative abundance was not confirmed by GLMM (Table S14).

Interestingly stable isotope values did not explain variation in beta-diversity in the small intestine, cecum, or rectum of $A$. speciosus despite the significant difference of $\delta^{15} \mathrm{~N}$ values between natural and urban populations. However, this does not exclude the effect of diet as the proportion of fats, proteins, and carbohydrates that can have a profound effect on microbial community structure (David et al., 2014; Singh et al., 2017) could not be estimated as we did not identify specific food items. For example, higher relative abundance of Lactobacillus in the small intestine of urban A. speciosus could be explained by the elevated consumption of anthropogenic food with animal protein (Fig. 6, Table S11). Increased protein consumption has been shown to positively affectLactobacillus abundance (Zhu et al., 2016), although we were unable to confirm such a relationship using GLMM (Table S13).

Increased abundance of Lactobacillus within urban populations as compared to those outside of city limits has been reported in house sparrows in Europe (Teyssier et al., 2018, 2020) and water dragons in Australia (Littleford-Colquhoun et al., 2019). Why we did not see a similar increase in $M$. rufocanus is an important question that must be investigated further. The bacterial genus Lactobacilluscontains many probiotic species that provide a wide range of benefits to their host including reduction of intestinal inflammation (Liu et al., 2010) and regulation of immune system function (Schluter et al., 2020). This important genus may aid in the successful adaption of animals to the urban environment by helping protect them from the adverse effects of novel stressors thereby allowing the animals to remain healthy.

Curiously, there was lower abundance of Helicobacter in the small intestine, but higher abundance in the colon of $M$. rufocanus in the urban parks as compared to those in the national forest (Fig. 6). The differential abundance was confirmed by GLMM in the small intestine and was nearly significant in the colon though neither were associated with diet (Table S12). Most species of Helicobacter thrive within the low $\mathrm{pH}$ environment of the stomach and small intestine, and many species are pathogenic in both humans and animals (On et al., 2015). Those species that have been isolated from the lower GIT such as H. hepaticus are known to induce inflammatory bowel disease (IBD) in immunocompromised animals and is associated with a markedly different microbial community of the cecum and colon (Yang et al., 2013). Although we did not test immune system function directly, there was less inter-individual variation in the gut microbiome of the small intestine within the urban populations due to species membership (Fig. 5, Table S8). The small intestine is the most immunologically active location in the entire body and the host immune system plays a pivotal role in shaping the gut microbial community (Bevins \& Salzman, 2011). Therefore, a similar and strong immunological shift in response to the urban environment due to elevated stress or pollution (Gao et al., 2018; Isaksson, 2015) could cause a convergence in the gut microbial community structure and leave them more susceptible to pathogens in the lower GIT such as Helicobacter. However, a lower body condition would be expected in diseased animals, yet we found no difference between urban and natural populations. Helicobacter may be a normal member of the gut microbial community of both rodent species as high abundance was also found throughout the lower GIT of $A$. speciosus in both habitat types (Fig. 6). Future studies should investigate species specific immune response to urbanization and how this may affect their gut microbiome and their susceptibility to pathogens.

Host species-specific response to urbanization

In comparing populations from urban and natural areas of northern Japan, we report host species-specific 
changes in the gut microbial communities of two sympatric species of rodent. Some of the changes may be associated with a dietary shift that is consistent with the two species' life history traits and may be influenced by the consumption of novel anthropogenic food resources or an altered rodent community structure increasing inter-specific competition. While we did not find a clear indication of dysbiosis in either species, the homogenization of the gut microbiome in the small intestine of $M$. rufocanus may be related to immunodeficiency and infection with a potentially pathogenicHelicobacter species in the lower GIT. On the other hand, $\operatorname{urban} A$. speciosus are harboring higher abundance of multiple probiotic genera that may protect them from the negative effects of ecosystem modification thereby allowing them to better adapt to disturbed habitats. Investigating changes in the gut microbiota in multiple host species and gut regions associated with the same degree of urbanization provides deeper insights into potential mechanisms behind such alterations that are associated with differing life histories. Understanding such mechanisms will help wildlife managers develop better protocols for maintaining healthy wildlife populations in our increasingly urbanized world.

\section{Acknowledgements}

We thank the members of the Koizumi lab who assisted in field surveys as well as those from the Hayakawa lab that helped in the initial preliminary DNA work. Thanks are given to Minoru Nakao and Mizuki Sasaki from the Department of Parasitology at Asahikawa Medical University for allowing us to utilize space and equipment within their lab during our field survey. We are indebted to Aiko Ohnuma from Hokkaido University Research Center for Zoonosis Control for her help conducting high throughput sequencing as well as Atsuko Sugimoto, Yumi Hoshino, and Aleksandr Nogovitcyn for their support in conducting stable isotope analysis. This study was supported by a Japanese Society for the Promotion of Science (JSPS) doctoral fellowhip (DC2, grant number:19J13514) and JSPS KAKENHI (grant numbers: 19H03118, 19F19097 and 19K16241)

\section{References}

Amato, K. R., Yeoman, C. J., Kent, A., Righini, N., Carbonero, F., Estrada, A., .. Leigh, S. R. (2013). Habitat degradation impacts black howler monkey (Alouatta pigra) gastrointestinal microbiomes. ISME Journal , 7 (7), 1344-1353. doi: 10.1038/ismej.2013.16

Baltensperger, A. P., Huettmann, F., Hagelin, J. C., \& Welker, J. M. (2015). Quantifying trophic niche spaces of small mammals using stable isotopes $\left(\delta^{15} \mathrm{~N}\right.$ and $\left.\delta^{13} \mathrm{C}\right)$ at two scales across Alaska. Canadian Journal of Zoology , 93 (7), 579-588. doi: 10.1139/cjz-2015-0025

Bateman, P. W., \& Fleming, P. a. (2014). Does human pedestrian behaviour influence risk assessment in a successful mammal urban adapter? Journal of Zoology , 93-98. doi: 10.1111/jzo.12156

Ben-David, M., \& Flaherty, E. A. (2012). Stable isotopes in mammalian research:a beginner's guide. Journal of Mammology , 93 (2), 312-328. doi: 10.1644/11-MAMM-S-166.1

Beninde, J., Veith, M., \& Hochkirch, A. (2015). Biodiversity in cities needs space: A meta-analysis of factors determining intra-urban biodiversity variation. Ecology Letters , 18 (6), 581-592. doi: 10.1111/ele.12427

Bevins, C. L., \& Salzman, N. H. (2011). Paneth cells, antimicrobial peptides and maintenance of intestinal homeostasis. Nature Reviews Microbiology , 9 (5), 356-368. doi: 10.1038/nrmicro2546

Boesmans, L., Valles-Colomer, M., Wang, J., Eeckhaut, V., Falony, G., Ducatelle, R., .. V Verbeke, K. (2018). Butyrate producers as potential next-generation probiotics: Safety assessment of the administration of $B u$ tyricicoccus pullicaecorum to healthy volunteers. MSystems , 3 (6), 1-12. doi: 10.1128/msystems.00094-18

Bokulich, N. A., Kaehler, B. D., Rideout, J. R., Dillon, M., Bolyen, E., Knight, R., .. Gregory Caporaso, J. (2018). Optimizing taxonomic classification of marker-gene amplicon sequences with QIIME 2's q2-featureclassifier plugin. Microbiome , 6 (1), 1-17. doi: 10.1186/s40168-018-0470-z

Bolyen, E., Rideout, J. R., Dillon, M. R., Bokulich, N. A., Abnet, C. C., Al-Ghalith, G. A., .. Caporaso, 
J. G. (2019). Reproducible, interactive, scalable and extensible microbiome data science using QIIME 2. Nature Biotechnology , 37 (8), 852-857. doi: 10.1038/s41587-019-0209-9

Bradley, C. A., \& Altizer, S. (2006). Urbanization and the ecology of wildlife diseases. Trends in Ecology and Evolution, 22 (2), 95-102. doi: 10.1016/j.tree.2006.11.001

Callahan, B. J., McMurdie, P. J., Rosen, M. J., Han, A. W., Johnson, A. J. A., \& Holmes, S. P. (2016). DADA2: High-resolution sample inference from Illumina amplicon data. Nature Methods , 13 (7), 581-583. doi: $10.1038 /$ nmeth.3869

Carmody, R. N., Gerber, G. K., Luevano, J. M., Gatti, D. M., Somes, L., Svenson, K. L., \& Turnbaugh, P. J. (2015). Diet dominates host genotype in shaping the murine gut microbiota. Cell Host and Microbe ,17 (1), 72-84. doi: 10.1016/j.chom.2014.11.010

Chin, E. Y., Dangler, C. A., Fox, J. G., \& Schauer, D. B. (2000).Helicobacter hepaticus infection triggers inflammatory bowel disease in T cell receptor $\alpha \beta$ mutant mice. Comparative Medicine ,50 (6), 586-594.

David, L. A., Maurice, C. F., Carmody, R. N., Gootenberg, D. B., Button, J. E., Wolfe, B. E., .. Turnbaugh, P. J. (2014). Diet rapidly and reproducibly alters the human gut microbiome. Nature , 505 , 559-563. doi: $10.1038 /$ nature 12820

Davis, N. M., Proctor, Di. M., Holmes, S. P., Relman, D. A., \& Callahan, B. J. (2018). Simple statistical identification and removal of contaminant sequences in marker-gene and metagenomics data.Microbiome , 6 (1), 1-14. doi: 10.1186/s40168-018-0605-2

de Paiva, C. S., Jones, D. B., Stern, M. E., Bian, F., Moore, Q. L., Corbiere, S., ... Pflugfelder, S. C. (2016). Altered mucosal microbiome diversity and disease severity in Sjögren syndrome.Scientific Reports , 6 , 1-11.doi: 10.1038/srep23561

Destoumieux-Garzón, D., Mavingui, P., Boetsch, G., Boissier, J., Darriet, F., Duboz, P., ... Voituron, Y. (2018). The one health concept: 10 years old and a long road ahead. Frontiers in Veterinary Science , 5 , 1-13. doi: 10.3389/fvets.2018.00014

Ditchkoff, S. S., Saalfeld, S. T., \& Gibson, C. J. (2006). Animal behavior in urban ecosystems: Modifications due to human-induced stress. Urban Ecosystems , 9 (1), 5-12. doi: 10.1007/s11252-006-3262-3

Donaldson, G. P., Lee, S. M., \& Mazmanian, S. K. (2016). Gut biogeography of the bacterial microbiota. Nature Reviews Microbiology, 14 (1), 20-32. doi: 10.1038/nrmicro3552

Faeth, S. H., Bang, C., \& Saari, S. (2011). Urban biodiversity: Patterns and mechanisms. Annals of the New York Academy of Sciences , 1223 (1), 69-81. doi: 10.1111/j.1749-6632.2010.05925.x

Fraune, S., \& Bosch, T. C. G. (2010). Why bacteria matter in animal development and evolution. BioEssays , 32 (7), 571-580. doi: 10.1002/bies.200900192

Fuirst, M., Veit, R., Hahn, M., Dheilly, N., \& Thorne, L. (2018). Effects of urbanization on the foraging ecology and microbiota of the generalist seabird Larus argentatus . PLos ONE . doi: 10.1371/journal.pone.0209200

Fujisawa, T., Shinohara, K., Kishimoto, Y., \& Terada, A. (2006). Effect of miso soup containing Natto on the composition and metabolic activity of the human faecal flora. Microbial Ecology in Health and Disease , 18 (2), 79-84. doi: 10.1080/08910600600931942

Gao, X., Cao, Q., Cheng, Y., Zhao, D., Yang, H., Wu, Q., .. Bian, J. (2018). Chronic stress promotes colitis by disturbing the gut microbiota and triggering immune system response. Proceedings of the National Academy of Sciences of the United States of America ,115 (13), E2960-E2969. doi: 10.1073/pnas.1806622115

Güneralp, B., Reba, M., Hales, B. U., Wentz, E. A., \& Seto, K. C. (2020). Trends in urban land expansion, density, and land transitions from 1970 to 2010: A global synthesis. Environmental Research Letters , 15 (4). doi: 10.1088/1748-9326/ab6669 
Han, X., Geller, B., Moniz, K., Das, P., Chippindale, A. K., \& Walker, V. K. (2014). Monitoring the developmental impact of copper and silver nanoparticle exposure in Drosophila and their microbiomes.Science of the Total Environment , 487 (1), 822-829. doi: 10.1016/j.scitotenv.2013.12.129

Hayakawa, T., Sawada, A., Tanabe, A. S., Fukuda, S., Kishida, T., Kurihara, Y., ... Agata, K. (2018). Improving the standards for gut microbiome analysis of fecal samples: insights from the field biology of Japanese macaques on Yakushima island. Primates , 59 (5), 423-436. doi: 10.1007/s10329-018-0671-x

Hooper, L. V., Midwedt, T., \& Gordon, J. I. (2002). How host-microbial interactions shape the nutrient environment of the mammalian intestine.Annual Review of Nutrition , 22 , 283-307. doi: 10.1146/annurev.nutr.22.011602.092259

Ims, R. A. (1987). Responses in spatial organization and behaviour to manipulations of the food resource in the vole Clethrionomys rufocanus . Journal of Animal Ecology , 56 (2), 585-596. doi: https://doi.org/10.2307/5070

Isaksson, C. (2015). Urbanization, oxidative stress and inflammation: A question of evolving, acclimatizing or coping with urban environmental stress. Functional Ecology , 29 (7), 913-923. doi: 10.1111/1365-2435.12477

Jackson, A. L., Inger, R., Parnell, A. C., \& Bearhop, S. (2011). Comparing isotopic niche widths among and within communities: SIBER - stable isotope bayesian ellipses in R. Journal of Animal Ecology ,80 (3), 595-602. doi: 10.1111/j.1365-2656.2011.01806.x

Kaneko, Y., Nakata, K., Saitoh, T., Stenseth, N., \& Bjørnstad, O. (1998). The biology of the vole Clethrionomys rufocanus : A review. Researches on Population Ecology , 40 (1), 21-37. doi: 10.1007/BF02765219

Klindworth, A., Pruesse, E., Schweer, T., Peplies, J., Quast, C., Horn, M., \& Glöckner, F. O. (2013). Evaluation of general 16S ribosomal RNA gene PCR primers for classical and next-generation sequencing-based diversity studies. Nucleic Acids Research , 41 (1), 1-11. doi: 10.1093/nar/gks808

Kohl, K. D., Dearing, M. D., \& Bordenstein, S. R. (2018). Microbial communities exhibit host species distinguishability and phylosymbiosis along the length of the gastrointestinal tract. Molecular Ecology , 27 (8), 1874-1883. doi: 10.1111/mec.14460

Labocha, M. K., Schutz, H., \& Hayes, J. P. (2014). Which body condition index is best? Oikos , 123 (1), 111-119. doi: 10.1111/j.1600-0706.2013.00755.x

Larson, R. N., Brown, J. L., Karels, T., \& Riley, S. P. D. (2020). Effects of urbanization on resource use and individual specialization in coyotes (Canis latrans ) in southern California. PLoS ONE ,15 (2), 1-23. doi: 10.1371/journal.pone.0228881

Littleford-Colquhoun, B. L., Frere, C. H., Weyrich, L. S., \& Kent, N. (2019). City life alters the gut microbiome and stable isotope profiling of the eastern water dragon (Intellagama lesueurii).Molecular Ecology , 28 , 4592-4607. doi: 10.1111/mec.15240

Liu, Y., Fatheree, N. Y., Mangalat, N., \& Rhoads, J. M. (2010). Human-derived probiotic Lactobacillus reuteri strains differentially reduce intestinal inflammation. American Journal of Physiology - Gastrointestinal and Liver Physiology , 299 (5), 1087-1096. doi: 10.1152/ajpgi.00124.2010

Logan, A. C., Jacka, F. N., \& Prescott, S. L. (2016). Immune-microbiota interactions: Dysbiosis as a global health issue. Current Allergy and Asthma Reports , 16 (2), 1-9. doi: 10.1007/s11882-015-0590-5

Long, E. S., Sweitzer, R. A., Diefenbach, D. R., \& Ben-David, M. (2005). Controlling for anthropogenically induced atmospheric variation in stable carbon isotope studies. Oecologia , 146 (1), 148-156. doi: 10.1007/s00442-005-0181-6

Mackenstedt, U., Jenkins, D., \& Romig, T. (2014). The role of wildlife in the transmission of parasitic zoonoses in peri-urban and urban areas.International Journal for Parasitology: Parasites and Wildlife , 4 (1), 71-79. doi: 10.1016/j.ijppaw.2015.01.006 
Matsubayashi, J., Morimoto, J., Mano, T., Aryal, A., \& Nakamura, F. (2014). Using stable isotopes to understand the feeding ecology of the Hokkaido brown bear (Ursus arctos ) in Japan. Ursus ,25 (2), 8797.doi: 10.2192/URSUS-D-12-00015.1

McMurdie, P. J., \& Holmes, S. (2013). Phyloseq: An R package for reproducible interactive analysis and graphics of microbiome census data. PLoS ONE , 8 (4). doi: 10.1371/journal.pone.0061217

Meddah, A. T. T., Yazourh, A., Desmet, I., Risbourg, B., Verstraete, W., \& Romond, M. B. (2001). The regulatory effects of whey retentate fromBifidobacteria fermented milk on the microbiota of the simulator of the human intestinal microbial ecosystem (SHIME). Journal of Applied Microbiology , 91 (6), 1110-1117. doi: $10.1046 / \mathrm{j} \cdot 1365-2672.2001 .01482 . \mathrm{x}$

Murray, M. H., Lankau, E. W., Kidd, A. D., Welch, C. N., Ellison, T., Adams, H. C.,... Hernandez, S. M. (2020). Gut microbiome shifts with urbanization and potentially facilitates a zoonotic pathogen in a wading bird. PLos ONE , 15 (3). doi: 10.1371/journal.pone.0220926

Murray, Maureen, Edwards, M. A., Abercrombie, B., Cassady, C., \& Clair, S. (2015). Poor health is associated with use of anthropogenic resources in an urban carnivore. Proceedings of the Royal Society B: Biological Sciences , 282 : 20150009. doi: 10.1098/rspb.2015.0009

Murray, MH, Sánchez, C., Becker, D., Byers, K., Worsley-Tonks, K., \& Craft, M. (2019). City sicker? A metaanalysis of wildlife health and urbanization. Frontiers in Ecology and the Environment ,17 (10), 575-583. doi: $10.1002 /$ fee. 2126

Oka, T. (1992). Home range and mating system of two sympatric field mouse species, Apodemus speciosus and Apodemus argenteus .Ecological Research , 7 (2), 163-169. doi: 10.1007/BF02348495

Oksanen, J., Blanchet, G., Friendly, M., Kindt, R., Legendre, P., McGlinn, P. R., .. Wagner, H. (2020). vegan: Community Ecology Package. $R$ package version 2.5-7, https://CRAN.R-project.org/package=Vegan

On, S. L. W., Lee, A., O'Rourke, J. L., Dewhirst, F. E., Paster, B. J., Fox, J. G., \& Vandamme, P. (2015). Helicobacter. Bergey's Manual of Systematics of Archaea and Bacteria . doi: 10.1002/9781118960608.gbm01073

Osaki, A., Sashika, M., Abe, G., Shinjo, K., Fujimoto, A., Nakai, M., ... Tsubota, T. (2019). Comparison of feeding habits and habitat use between invasive raccoons and native raccoon dogs in Hokkaido, Japan. BMC Ecology , 19 (1), 1-15. doi: 10.1186/s12898-019-0249-5

Owens, A. C. S., Cochard, P., Durrant, J., Farnworth, B., Perkin, E. K., \& Seymoure, B. (2020). Light pollution is a driver of insect declines.Biological Conservation, 241 , 108259. doi: 10.1016/j.biocon.2019.108259

Pagani-Núñez, E., Liang, D., He, C., Zhou, X., Luo, X., Liu, Y., \& Goodale, E. (2019). Niches in the Anthropocene: passerine assemblages show niche expansion from natural to urban habitats. Ecography ,42 (8), 1360-1369. doi: 10.1111/ecog.04203

Parnell, A. (2020). simmr: A stable Isotope mixing model. $R$ Package . https:/CRAN.Rproject.org/package $=$ simmr

Phillips, J. N., Berlow, M., \& Derryberry, E. P. (2018). The effects of landscape urbanization on the gut microbiome: An exploration into the gut of urban and rural white-crowned sparrows. Frontiers in Ecology and Evolution , 6 , 148. doi: 10.3389/fevo.2018.00148

Pinheiro, J., Bates, D., Debroy, S., \& Sarkar, S. (2020). nlme: Linear and nonlinear mixed effects models. $R$ Package. https://CRAN.R-project.org/package=nlme

Plummer, M. M. (2019). rjags: Bayesian graphical models using MCMCR.R Package version 4-10. https://CRAN.R-project.org/package=rjags

Price, M. N., Dehal, P. S., \& Arkin, A. P. (2010). FastTree 2 - Approximately maximum-likelihood trees for large alignments. PLoS ONE , 5 (3). doi: 10.1371/journal.pone.0009490 
Saitoh, T., Osawa, J., Takanishi, T., Hayakashi, S., Ohmori, M., Morita, T., .. Maekawa, K. (2007). Effects of acorn masting on population dynamics of three forest-dwelling rodent species in Hokkaido, Japan.Population Ecology , 49 (3), 249-256. doi: 10.1007/s10144-007-0041-9

Sato, J. J., Kawakami, T., Tasaka, Y., Tamenishi, M., \& Yamaguchi, Y. (2014). A few decades of habitat fragmentation has reduced population genetic diversity: A case study of landscape genetics of the large Japanese field mouse, Apodemus speciosus . Mammal Study,39 (1), 1-10.doi: 10.3106/041.039.0102

Schluter, J., Peled, J. U., Taylor, B. P., Markey, K. A., Smith, M., Taur, Y., .. Xavier, J. B. (2020). The gut microbiota is associated with immune cell dynamics in humans. Nature , 588, 303-307. doi: 10.1038/s41586020-2971-8

Segata, N., Izard, J., Waldron, L., Gevers, D., Miropolsky, L., Garrett, W. S., \& Huttenhower, C. (2011). Metagenomic biomarker discovery and explanation. Genome Biology , 12:R60 . doi: 10.1186/gb-2011-12-6r60

Seto, K. C., Güneralp, B., \& Hutyra, L. R. (2012). Global forecasts of urban expansion to 2030 and direct impacts on biodiversity and carbon pools. Proceedings of the National Academy of Sciences of the United States of America , 109 (40), 16083-16088. doi: 10.1073/pnas.1211658109

Shochat, E., Warren, P. S., Faeth, S. H., McIntyre, N. E., \& Hope, D. (2006). From patterns to emerging processes in mechanistic urban ecology. Trends in Ecology and Evolution , 21 (4), 186-191.doi: 10.1016/j.tree.2005.11.019

Singh, R. K., Chang, H. W., Yan, D., Lee, K. M., Ucmak, D., Wong, K., .. Liao, W. (2017). Influence of diet on the gut microbiome and implications for human health. Journal of Translational Medicine ,15 (1), 1-17. doi: 10.1186/s12967-017-1175-y

Sugden, S., Sanderson, D., Ford, K., Stein, L. Y., \& St. Clair, C. C. (2020). An altered microbiome in urban coyotes mediates relationships between anthropogenic diet and poor health. Scientific Reports ,10 (1), 1-14. doi: 10.1038/s41598-020-78891-1

Suzuki, T. A., Martins, F. M., \& Nachman, M. W. (2019). Altitudinal variation of the gut microbiota in wild house mice. Molecular Ecology , 28 , 2378-2390. doi: 10.1111/mec.14905

Tatsukawa, K., \& Murakami, O. (1976). On the food utilization of the Japanese wood mouse Apodemus speciosus (Mammalia: Murideae).Physiology and Ecology of Japan , 17, 133-144.

Team, C. R. (2020). R: A language and environment for statistical computing. $R$ Foundation for Statistical Computing . https://www.r-project.org/

Teyssier, A., Matthysen, E., Hudin, N. S., de Neve, L., White, J., \& Lens, L. (2020). Diet contributes to urban-induced alterations in gut microbiota: Experimental evidence from a wild passerine.Proceedings of the Royal Society B: Biological Sciences ,287 (1920). doi: 10.1098/rspb.2019.2182

Teyssier, A., Oscar, L., Saleh, N., Strubbe, D., Matthysen, E., Lens, L., \& White, J. (2018). Inside the guts of the city: Urban-induced alterations of the gut microbiota in a wild passerine. Science of the Total Environment , 612 , 1276-1286. doi: 10.1016/j.scitotenv.2017.09.035.

Yang, I., Eibach, D., Kops, F., Brenneke, B., Woltemate, S., Schulze, J., .. Suerbaum, S. (2013). Intestinal microbiota composition of interleukin-10 deficient C57BL/6J mice and susceptibility toHelicobacter hepaticus -induced colitis. PLoS ONE ,8 (8). doi: 10.1371/journal.pone.0070783

Yoon, T. J., Kim, D. G., Kim, S. Y., Jo, S. Il, \& Bae, Y. J. (2010). Light-attraction flight of the giant water bug, Lethocerus deyrolli (Hemiptera: Belostomatidae), an endangered wetland insect in East Asia. Aquatic Insects , 32 (3), 195-203. doi: 10.1080/01650424.2010.508045

Zhang, X., Pei, Y. F., Zhang, L., Guo, B., Pendegraft, A. H., Zhuang, W., \& Yi, N. (2018). Negative binomial mixed models for analyzing longitudinal microbiome data. Frontiers in Microbiology ,9 , 1-10. doi: 
10.3389/fmicb.2018.01683

Zhu, Y., Lin, X., Li, H., Li, Y., Shi, X., Zhao, F., .. Zhou, G. (2016). Intake of meat proteins substantially increased the relative abundance of genus Lactobacillus in rat feces. PLoS ONE ,11 (4). doi: 10.1371/journal.pone. 0152678

\section{Author Contributions}

JLA and IK conceived and designed the study with input from MAMM, RN, and TH. JLA conducted the field surveys and sample processing with support from AMM, MAMM, RN, and TH. JLA, MAMM, and WMA analyzed the microbiome data and created the associated figures. AMM analyzed the stable isotope data and created the relevant figures. All authors participated in the discussion of the results and made contributions to the final manuscript.

\section{Data accessibility statement}

The raw $16 \mathrm{~S}$ amplicon sequences have been submitted to the DNA database of Japan (DDBJ: https://www.ddbj.nig.ac.jp/index-e.html) under the accession numbers DRA011343, DRA011772.

Table 1 : The average proportion of each food item in the diet of $A$. speciosus and M. rufocanus in natural and urban populations (percent \pm standard deviation).

Figure 1: Comparison of a) $\delta^{13} \mathrm{C}$ and b) $\delta^{15} \mathrm{~N}$ between species and populations in urban and natural areas. The $p$-values are based on pairwise Wilcoxon rank-sum test.

Figure 2: Total area convex hulls (solid lines) and 95\% confidence intervals around bivariate means (filled ovals) for natural (green) and urban (gray) populations of A. speciosus and M. rufocanus as calculated in SIBER.

Figure 3: Alpha diversity along the gastrointestinal tract of $A$. speciosus and $M$. rufocanus in natural and urban populations according to a) Shannon's diversity and b) Faith's PD. The black dashed line separates host species and the grey dashed line separates habitat type.

Figure 4: PCoA plots of the gut microbial community along the gastrointestinal tract of $A$. speciosus based on unweighted and weighted UniFrac dissimilarity metrics. Blue are individuals from the natural areas and red are urban.

Figure 5: PCoA plots of the gut microbial community along the gastrointestinal tract of M. rufocanus based on unweighted and weighted UniFrac dissimilarity metrics. Blue are individuals from the natural areas and red are urban.

Figure 6: Relative abundance of four genera of interest in each gut region in each host species from natural and urban habitats. SI is the small intestine, CE the cecum, CL the colon, and RC the rectum.

\section{Hosted file}

Table 1.docx available at https://authorea.com/users/428057/articles/532061-dietary-nichebreadth-influences-the-effects-of-urbanization-on-the-gut-microbiota-of-sympatricrodents 

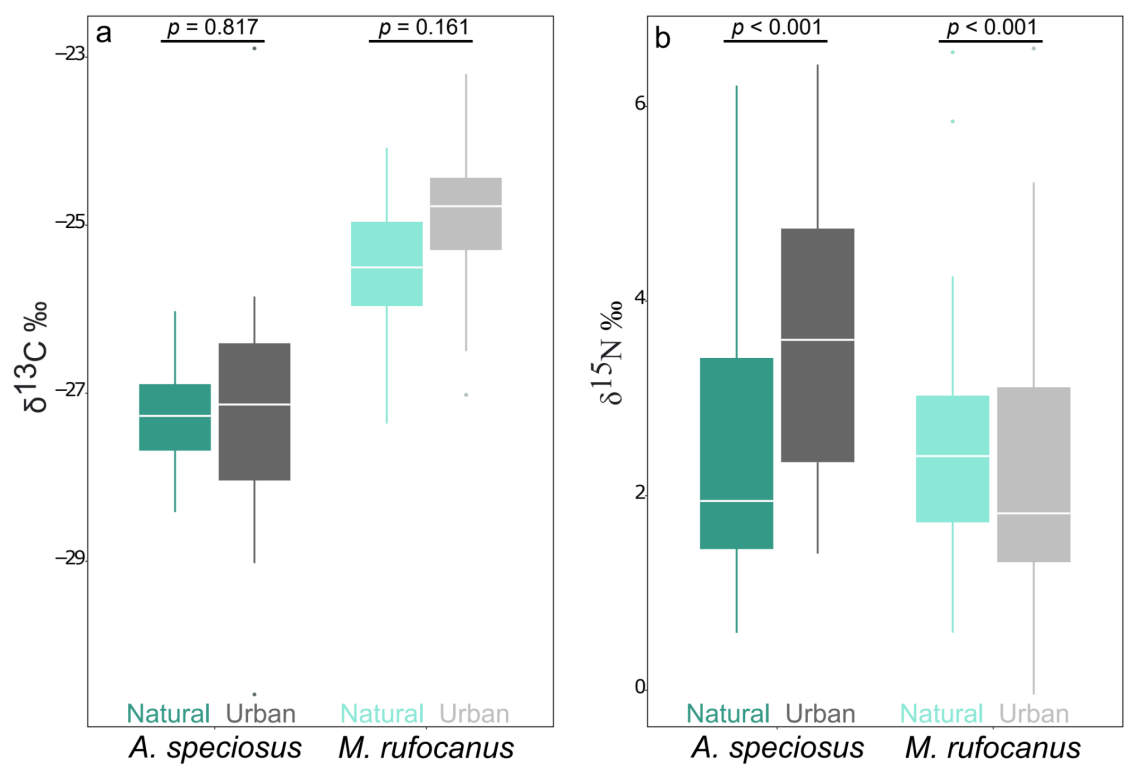


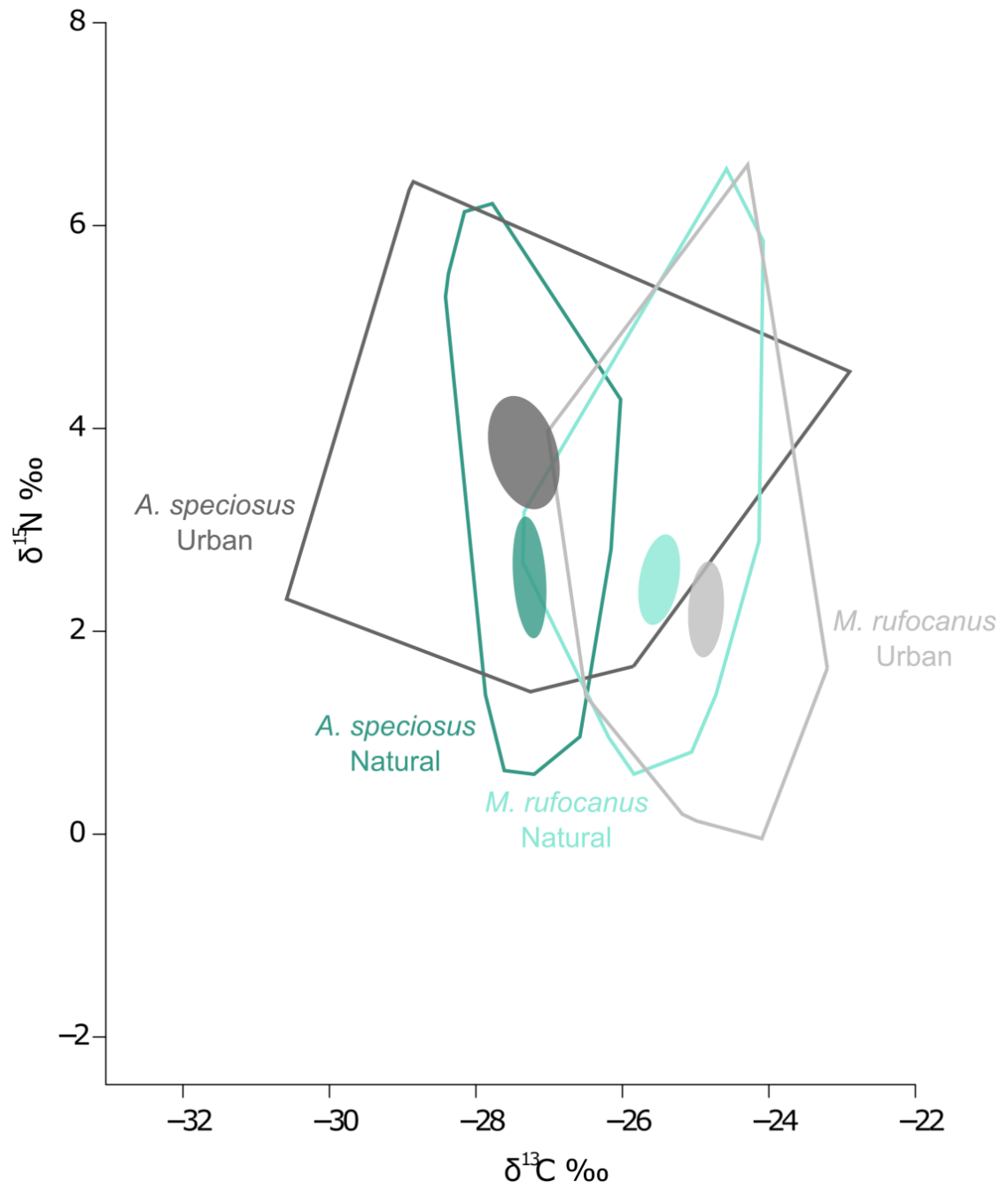




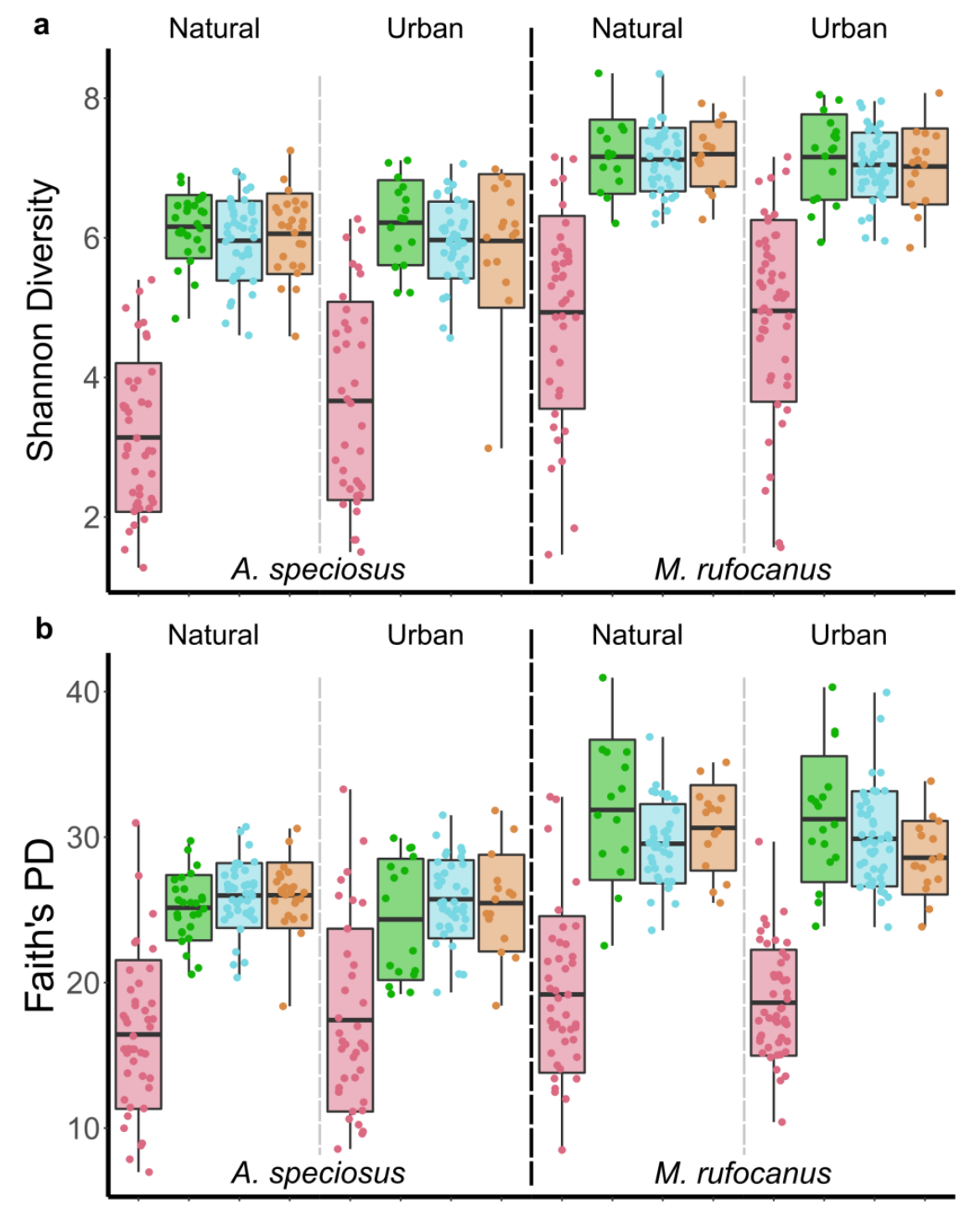

Gut Region: 兒 Small Intestine 夏 Cecum 白 Colon 审 Rectum

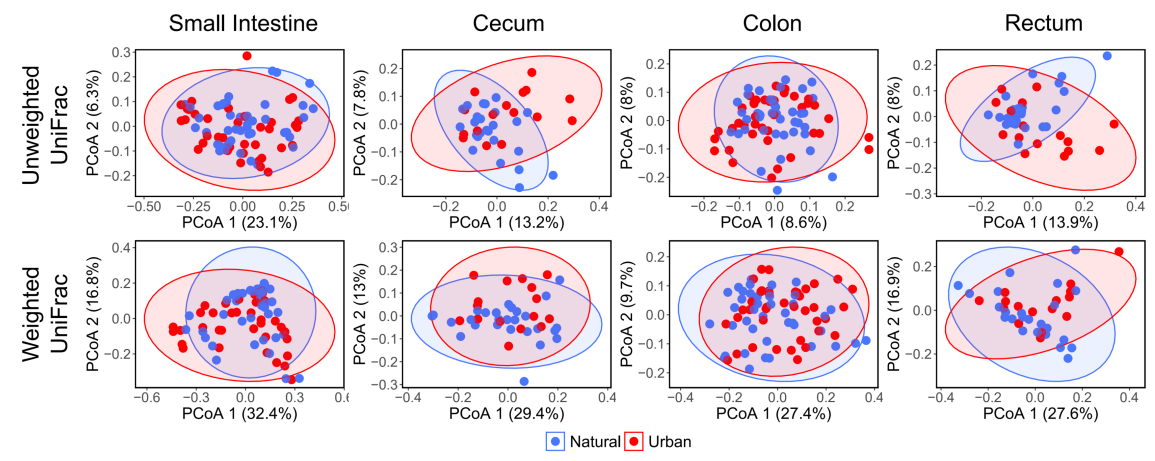



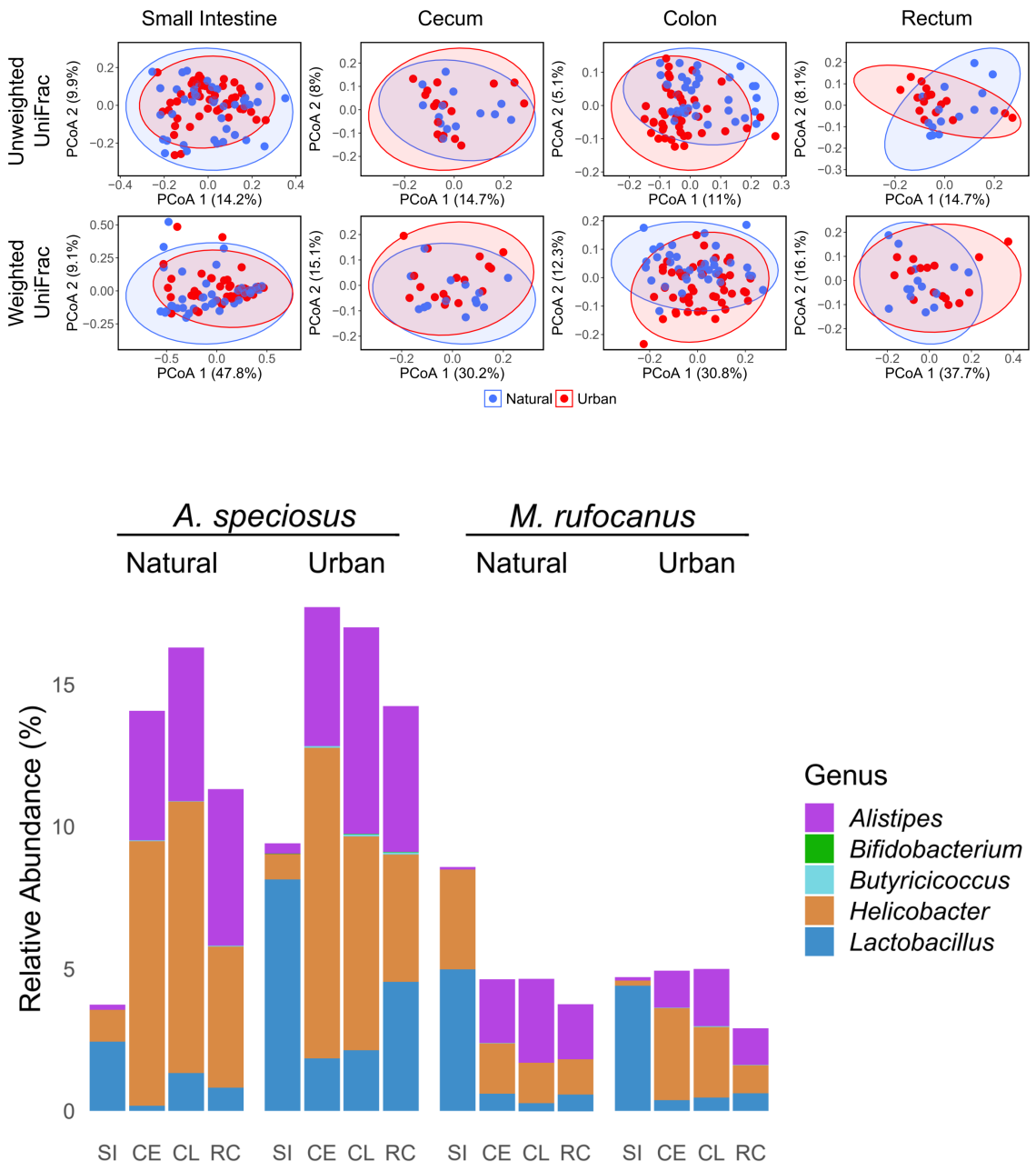